\title{
HIGHLIGHTS
}

\section{Prediction of hypocalcemia: is phosphate the answer?}

A serum phosphate test could provide a cheap and rapid means of predicting post-thyroidectomy hypocalcemia in vitamin-D-replete patients, report researchers.

Temporary hypocalcemia is a major cause of delayed hospital discharge following thyroidectomy. Biomarkers currently used in the prediction of this postoperative complication include calcium and parathyroid hormone (PTH), but they are not without their limitations. "None is ideal either due to reliability or cost," stresses senior author Fausto Palazzo of Imperial College London. "The idea for the current study was a casual observation that changes in serum phosphate seemed to precede hypocalcemia."

Thyroidectomy can impair secretion of PTH by the parathyroid glands, which results in decreased circulating levels of the hormone. The connection with phosphate arises because serum phosphate levels rapidly increase after a decrease in circulating PTH levels.
44 ...phosphate levels predicted hypocalcemia in ... patients who were vitamin D replete... 77

To examine the value of serum phosphate as a possible biomarker for the prediction of temporary hypocalcemia, the researchers retrospectively assessed the medical records of 111 consecutive patients who had undergone thyroidectomy; only patients with sufficient data on serum calcium, phosphate, PTH and 25-hydroxyvitamin D levels were included.

Hypocalcemia that required treatment developed in 29 patients from postoperative day 1 onwards. Of these patients, serum phosphate levels predicted hypocalcemia in all 19 patients who were vitamin $D$ replete (25-hydroxyvitamin D $>25 \mathrm{nmol} / \mathrm{l}$ ). In these 19 patients, mean serum phosphate levels rose from the evening of surgery to the following morning, whereas in patients who did not develop hypocalcemia, levels had fallen by postoperative day 1 -consistent with normal diurnal variation in serum phosphate levels. The biomarker was not a reliable means of predicting hypocalcemia in patients with 25-hydroxyvitamin D levels of $<25 \mathrm{nmol} / \mathrm{l}$ because vitamin $\mathrm{D}$ deficiency affects serum phosphate levels. This limitation of serum phosphate measurement might be rectified by vitamin D supplementation before surgery to normalize patients' vitamin D levels.

Early identification and treatment of patients at risk of hypocalcemia is not the only aim of improved prediction, as Palazzo points out. "With the increasing drive towards early discharge, the clinical implications are significant: this is a reliable predictor of hypocalcemia that unlike PTH is universally available and very cheap-clearly an advantage in the current economic climate."

\section{Carol Wilson}

Original article Sam, A. H. et al. Serum phosphate predicts temporary hypocalcaemia following thyroidectomy. Clin. Endocrinol. (Oxf) doi:10.1111/j.1365-2265.2010.03949.x 